\title{
III. \\ Zum Problem der Gegenstandssetzung der Philosophiegeschichte. \\ Von
}

Dr. David Einhorn, Wien.

Wie die Philosophie ihr Suchen mit dem Suchen nach dem Gegenstande der Philosophie beginnen muß, so muß auch die Philosophiegeschichte im Gegensatz $\mathrm{zu}$ fast allen anderen erkenntnisgeschichtlichen Untersuchungen die Bestimmung ihres Gegenstandes überhaupt als ihr erstes und vielleicht eines der ernstesten Probleme behandeln. In bezug auf die Philosophie sind wir uns allgemein einer äußerst merkwürdigien Freiheit bewußt, ihren Gegenstand in dieser oder jener Weise bestimmen zu können: sei es im Anschluß an irgendwelche Überlieferung oder eine zeitgenössische Auffassung die erste Entscheidung über Sinn und Sein der Philosophie zu treffen, sei es auf einem selbsteigenen Wege neue Wirklichkeiten als die wahren Erkenntnisgebiete der Philosophie hinzustellen. Diese Freiheit bezw. die ihr entsprechendie wunderbare Wandlungsfähigkeit des Gegenstandes der Philosophie im Laufe der Zeiten unterscheidiet die letztere schlechterdings von aller empirischen, d. h. an einen bestimmten Gregenstand unabänderlich gebundenen Wissenschaft - wie dies bereits und wohl zu allererst Hegel genau gesehen und hervorgehoben hatte. Denn es wäre doch ganz ungereimt, wenn jemand allen Ernstes behaupten wollte, daß eine Erfahrungswissenschaft, wie etwa die Botanik oder die Mineralogie ihre althergebrachiten Forschungsobjekte aufgeben und ganz andere Bezirke der Gesamtwirklichkeit, etwa die Bewegungen der Himmelskörper od'er die Grundformen des religiösen Erlebnisses für sich eines Tages in Anspruch nehmen könnten. Dile Wissenschaft zeïchnet uns ihren 
Gegenstand vor. In der Philosophie sind wir es, die dem Erkennen seinen Gegenstand aus freien Stücken setzen. Indem nun differente Forscher auf differenten mehr oder weniger selbständigen Wegen von dieser ihrer wunderbaren Freiheit Gebrauch mächen, entsteht jenes gewaltige Chaos dier bisherigen Gegenstands-Setzungen der Philosophie, jenes so häufig geschilderte und als leider ganz unheilbares Übel beklagte- bellum omnium contra omnes, das seinesgleichen im Bereiche des menschlichen Erkennens vergeblich suchen würde.

So ist es mit der Philosophie. Weshalb soll es aber mit der Gegenstands-Setzung der Philosophiegeschichte irgendwelche Schwierigkeiten geben? Wie die Geschichte im allgemeinen, so kann doch auch die Philosophiegeschichte im besonderen ganz unmöglich ein Freies sein! Mag die Philosophie in ider ersten Stunde ihres Schaffens wohl noch ein proteusartiges Ding, ein rätselhaftes Spiel von verborgenen Möglichkeiten, ein Freies sein, allein als ge s chaffene Philosophie, als Teil der Geschichte der Philosophieist sie ja eine ebenso gebundene, unfreie, empirische Tatsache wie etwa die fossilen Überreste eines Achäopterix eine unabänderlich gegebene-Tatsache der Paläontologie bilden. Die Geschichte, mag sie nun auch Philosophieg̀èschichte sein, ist und bleibt unter allen Umstänlen eine Vergangenheits-Setzung, und die Vergangenheit ist ia wahrlich kein Gebiet, das unserer Freiheit unterworfen wäre, kein Reich, an dem wir etwas zü ändiern vermögen. Die GegenstandsSetzung der Philosophiegeschichte soll folglich genau so. wenig Bedenken unterliegen wie etwa die Gegenstandis-Setzung der Geschichte der Zoologie oder der Physik. Wie kein Geschichtsschreiber der Zoologie eine andere Wirklichkeit zum Gegenstand der Zoologiegeschichte erheben kann als diejenige, in ider bereits seine Vorgänger das Objekt der Zoologiegeschichte erkannten, genau so wenig dürfte ein Philosophiehistoriker in betreff ' der Gegenstands-Setzung der Philosophiegeschichte von einem Vorgänger oder Mitarbeiter wesentlich abweichen können.

Wenn dies aber auch noch so einfach scheint und wenn der vorhergehenden Betrachtung des geschichtlichen Phänomens zufolge keine Möglichkeit des Auseinandergehens der Meinungen in" bezug aui die allgemeingültige Bestimmung des "Gegenstandes der Philosophiegreschichte $\mathrm{zu}$ bestehien scheint, so fehlt es doch nicht an ge- 
wichtigen Indizien, die ernste Zweifel an der Möglichkeit einer so glatten Lösung, wie sie uns im Vorstehenden zuwinkt, wachzurufen geeignet sind.

Ein derartiges Indizium bedeutet der im Nachstehenden angeführte überaus charakteristische Gedankengang, der die Stellungnahme Wundts zum Problem dier Gegenstands-Setzung der Philosophiegeschichte zum Ausdruck bringt.

Im ersten Aufsatz seiner Essays (1906) äußerst sich Wundt folgendermassen (S. 26-7):

„Daß neben der kritischen und systematischen die his torische Behandlung der philosophischen Probleme fortan ihre Bedeutung behält, bedarf kaum noch der besondieren Betonung. Doch werden die Folgen der veränderten Stellung, in welche die Philosophie selbst gelangt ist, sicherlich auch auf deren Geschichte ihre Wirkung äußern müssen. Je mehr sie aufhört, eine bloße Geschichte dier philosophischen Systeme zu.sein, um sich in eine-allgemeine Geschichte der Wissenschaft umzuwandeln, desto mehr wird sie eine fühlbare Lücke ausfüllen in dem Zusammenhiang unseres Wissens. Der wahre Beruf des Historikers der Philosophie ist es, nicht eine Chronik dier Meinungen und Verirrungen der Philosophen zu schreiben, sondern ein Bild der die Gesamtentwicklung der Wissenschait beherrschenden Ideen $z u$ 'entwerfen."

„Die zahlreichen Quellen dier Erkenntnis, die in iden verschiedenen Wissensgebieten fließen, hat so die Geschichte der Philosophie zu einem Strome zu sammeln, an welchem man zwar nicht den Verlauf jeder besonderen Quelle, wohl aber die Richtung wiedererkennt, die sie alle zusammen genommen haben. Dem Bewußtsein der jüngst vergangenen Zeit war diese Wechselwirkung zuweilen abhanden gekommen. Den einzelnen Wissenschaften entspringt daraus der geringere Vorwurf. Denn die Sache der Philosophie ist es, die gute Beziehung $\mathrm{zu}$ denselben lebendlig $\mathrm{zu}$ erhalten, indem sie ihnen entrehnt, was sie bedlarf, die Grundlage der Erfahrung, und ihnen mitteilt, was sie entbehren, 'den allgemeinen Zusammenhang der Erkenntnisse."

Diese Wundtsche Auffassung widierstreitet nun schnurstracks allien hergebrachten Bemühungen um eine Gegenstands-Bestimmung der Philosopiegieschichte-" Daß es "der wahre Beruf" der Philosophiegeschichte sei, nicht über die Erkenntnisse der Philosophie, 
oder wie Wundt etwas verächtlich meint die „Meinungen und Verirrungen der Philosophen zu schreiben", „sondern ein Bild der die Gesamtentwicklung. der Wissenschaft beherrschende Ideen $\mathrm{zu}$ entwerfen", will uns fast genau so paradox klingen, wie wenn jemand behaupten wollte, die Philosophiegeschichte solle eigentlich nicht die „Lehrmeinungen“ der Philosophen, sondern etwa der - Zoologen oder Theologen oder Astronomen darstellen. Alein wir müßten dann im letzteren Falle die unabweisbare Frage beantworten: warum soll denn die Geschichte der Meinungen der Zoologen oder Astronomen gerade Philosophiegeschichte und nicht etwa Zoologiegeschichte oder Astronomiegeschichte heißen? Mit welchem Rechte darf die Geschichte der die Gesamtentwicklung der Wissenschaft, beherrschenden Ideen Philosophiegeschichte und nicht einfach und natürlich prinzipielle Wissenschaftsgeschichte heißen? Und wie sollten wir dann obendrein jenes Gebiet der allgemeinen Erkenntnisgeschichte benennen, welches ein Bild dier Erkenntnisse dier Philosophen zu entwerfen sich angelegen sein läßt?! Soll dieses Gebiet etwa den Namen der Zoologiegeschichte oder der prinzipiellen Wissenschaftsgeschichte für sich in Anspruch nehmen? Eine Philosophiegeschichte, die sich a priori über die „Chronik der Meinungen und Verirrungen der Philosophen" verächtlich hinwegsetzt, die von ihr keinen Ausgangspunkt nehmen zu können vermeint, eine Philosophiegeschichte, die kurzum mit der Geschichte der philosophischen Erkenntnisse nichts gemeinsames hat, ist, eben gar keine Philosophiegeschichte. So muß zunächst bereits aus allgemeinen logischen Gründen der Wundtsche Begriff der Philosophiegeschichte, der den wahren Begriff augenscheinlich geradezu auf d'en Kopf stellt, schlechthin zurückgewiesen werden.

Die völlige. Unhaltbarkeit dieser Wundtschen Auffassung jst nun viel $z u$ auffallend, als daß bei einem so hervorragenden und überaus umsichtigen Forscher wie Wilhelm Wundt eine rein persönliche Fehlerquelle anzunehmen wäre. Es darf vielmehr von vornherein als durchaus wahrscheinlich gelten, daß ernste sachliche Motive den gefeierten Leipziger Denker mit dazu verleitet haben müssen, eine so sonderbare Ansicht in Sachen der Philosophiegeschichte auszusprechen. Indem wir nun in die anderweitigen Gedankengänge unseres Forschers des Näheren eindringen, insbesondere diejenigen, die die Beziehungen zwischen Philosophie und Wissenschaft be- 
handeln, stellt sich alsbald heraus, daß die so paradoxe und haltlose Auffassung auf eine dem ersten Anschein nach Jurchaus unangreifbare und landläufige Begründung zurückgeht.

Denn Wundt folgte iderjenigen logischen Notwendigkeit. die die Gegenstands-Setzung der Philosophiegeschichte aui die GegenstandsBestimmung der Philosophie selbst gründet. Und da ihm die Aufgabe der Philosophie im Gegensatz zu den zahllosen Auffassungen vieler anderer Forscher in ider Herstellung eines Zusammenhanges unserer allgemeinen wissenschaftlichen Erkenntnisse zu liegen scheint, so konnte ihm doch nichts anderes zur Aufgabe der Philosophiegeschichte werden als eben die Verfolgung und Darstellung aller bisherigen geschichtlichen Versuche, die jeweils die Gesamtwissenschaft beherrschenden Ideen in ihrem Zusammenhange zu erfassen. Ist es die Aufgabe der Philosophie eine Vereinheitlichung und Abrundung des wissenschaftlichen Weltbildies zu liefern, so kann auch nichts anderes Aufgabe der Philosophiegeschichte sein als die Geschichte aller derartigen Vereinheitlichungs- und Abrundungs-Versuche darzustellen. Und so scheint uns Wundts Ansicht, die in ihrer Behauptung so paradox, so haltlos klang, in ihrer Begründung doch nichts weniger als paradox und grundllos zin sein, - ein eigenartiger Sachverhalt, den es durchaus irgendwie aufzuhellen gilt.

Dem nächsten Anblick zufolge stehen wir vor einer regelrechten Antinomie: 1. Die Geschichte der Philosophie ist eine ge sch icht i i ch e Wissenschaft, eine Lehre von der Vergangenheit, mithin als solche für alle ihrem Gegenstande nach idaentisch und unabänderlich gegeben. 2. Die Geschichte der Philosophie ist aber zugleich eine Lehre von der bisherigen Entwicklung der $\mathrm{Phil}$ o s oph i e. Da jedoch eine eigene Bestimmung des Gegenstandes der Philos ophie jedem prinzipiell freisteht, so liegt damit notwendig auch die GegenstandsSetzung der Philos ophiegeschichte im Bereiche der Freiheit jedes einzelnen Forschers.

So scheint die Antinomie von Notwendigkeit und Freiheit, von Einheit und Vielheit augenscheinlich und ihre Schwierigkeit unaufheblich $\mathrm{zu}$ sein. Indes wenn wir tiefer in die letztere Bestimmung des Objekt der Philosophiegeschichte eindringen, erwachen doch Bedenken gegen ihre Geltung, die die Spannung unserer Antinomie rationeller $\mathrm{zu}$ giestalten vermögen. Zunächst muß man sich doch gegenwärtig halten, daß wer die Gegenstands-Setzung der Philo- 
sophiegeschichte nicht auch nach sämtlichen fremden GegenstandsSetzungen der Philosophie, sondern lediglich nach seiner eigenen bestimmt, der damit eigentlich.keine Geschichte der Philosophie schlechthin, sondern lediglich und alleịn eine Geschichte der Erkenntnisversuche in bezug auf seinen eigenen philosophischen Gegenstand im Auge behält - eine Privatgeschichte der Philosophie, die die gewaltige Weite und Freiheit fremder Philosophien und damit der Philosophie im allgemeinen keineswegs zu umspannen vermag. Die Erkenntnisgeschichte des eigenen philosophischen Gegenstandes läßt sich keineswegs mit der Erkenntnisgeschichte sämtlicher bis nun gesetzter philosophischer Gegenstände vergleichen und messen, sie ist sozusagen bodenständig, während in der Geschichte der Philosophie überhaupt der philosophische Gegenstand sich beständig wechselt und wandelt. Wie die $\mathrm{Ph}$ il os ophie überhaupt reicher und vielgestaltiger ist als ein e Philosophie, so ist die Geschichte der Philosophie unvergleichlich reicher als die Geschichte e iner Philosophie, d. h. die Erkenntnisgeschichte eines philosophischen Gegenstandes. Die Geschichte der Philosophie überhaupt ist die Geschichte der Freiheit der Philosophie, d. h. der Wandlungsfähigkeit des philosophischen Gegenstandes. Eine Privatgeschichte der Philosophie im oben dargeiegten Wundtschen Sinne ist eine Gieschichte der Versuche ein und denselben in starrer Gegebenheit umwandelbaren Gegenstand $z u$ erkennen.

Daraus erhellt bereits zur Genüge, daß nicht sowohl mit gleichem Recht beide Wege zur Gegenstands-Setzung ider Philosophiegeschichte eingeschlagen werden können, daß das völlig subjektive einseitige Verfahren Wundts tiefer eindringender Kritik schwerlich standzuhalten vermag.

Allerdings ist nicht bloß die auf eine rein subjektive Definition dies Gegenstandes der Philosophie gegründete Gegenstands-Setzung der Philosophiegeschichte - wie wir sie bei Wundt sehen - nicht - unbedenklich. Auch das rein objektive Verfahren zur exakten Bestimmung des Gegenstandes der philosophiegeschichtlichen Wissenschait, das die Geschichte, die Vergangenheit zur universellen und unverrückbaren Basis selber erhebt, mag es dem nächsten Anblick noch so selbstverständlich und unangreifbar scheinen, erweist sich beim Versuch einer näheren Durchführung als voller Schwierigkeiten und Verwicklungen, ja eine sichere Unmöglichkeit. Das sah scharf 
und betonte mit besonderem Nachdruck der Gegner Wundts, der Philosophiehistoriker W. Windelband in folgender vortrefilicher Formulierung (Präludien, Bd. I., S, $7-9$ ):

„Aus dieser Verschiedenheit der Gegenstände der Philosophie èrgibt sich nun eine nicht unerhebliche, prinzipiell bisher noch kaum behandelte Schwierigkeit für den Historiker, die Frage nämlich, in welcher Ausdehnung und in welchen Grenzen er die von einem Philosophen herrührenden Ansichten und Lehren, abgesehen von der biographischen Bedeutung, die sie zur Charakteristik seiner Persönlichkeit haben können, in die Geschichte der Philosophie aufnehmen soll. Nur zwei völlig konsequente Wege scheinen hier offen zu stehen: entweder man folgt der Geschichte selbst in alle Wunderlichkeiten ihrer Namengebung und läßt die historische Darstellung ganz ebenso wie das "philosophische“ Interesse von dem einen Gregenstand zu dem anderen wandern, oder man legt eine bestimmte Definition der Philosophie zugrunde und vollzieht nach dieser die Auswahl und die Ausscheidung der einzelnen Lehren. Im ersteren Falle erkauft man die „historische Objektivität" durch eine ver.wirrende Verschiedenartigkeit und Zusammenhanglosigkeit der Gegenstände; im anderen Fall beruht die Einheitlichkieit und Durchsichtlichkeit, welche erreicht wird, auf der Einseitigkeit, mit der man eine persönlich bestimmte Voraussetzung als Schema in die geschichtliche Bewegung hinein verlegt. Die meisten Historiker der Philosophie haben, ohne darüber Rechenschaft zu geben oder auch *wohl geben zu können, èinen Mittelweg eingeschlagen, indem sie solche Theorien der Philosophen, welche in das Detail der besonideren Wissenschaften eingreifen, nur in ihrem prinzipiellen Zusammenhang mit der Gesamtlehre entwickelt und auf die Reproduktion der speziellen Durchführung, je nach der Ausdehnung ihrer Arbeit, mehr oder minder verzichtet haben. $\mathrm{Da}$ jedoch dafür ein bestimmtes Kriterium nicht angegeben ist und auch nicht in selbstverständlich allgemein gültiger Weise angegeben werden kann, so hat an Stelle dessen meist die Willkürlichkeit des persönlichen Interesses oder die Zufälligkeit eines gewissen Taktgefühls treten müssen."

„Diese Schwierigkeit ist, wie die geschichtlichen Verhältnisse einmal liegen, prinzipiell in der Tat nicht zu heben."

Die Gegenstands-Setzung der Philosophiegeschichte kann deshalb unmöglich auf eine Gegenstands-Setzung der Philosophie ge- 
gründet werden, weil es keine Definition des Gegenstandes der Philosophie gibt, die nicht von bloß subjektiver Gültigkeit wäre und nicht bloß einen, sondern sämtliche wahrhaft philosophischen Gegenstände umfassen würde. Welche aber die wahrhaft philosophitschen Giegenstände sind, das wissen wir nun ebensowenig, da es eben noch keinen allgemeingültigen Maßstab gibt, der uns zur Unterscheidung der philosophischen Gegenstände in der Geschichte von den nichtphilosophischen Gegenständen in derselben verhelfen könnte. Wohl ist also die Wirklichkeit der Philosophiegeschichte in ider Vergangenheit unabänderlich festgelegt, allein fü $\mathrm{r}$ uns ist sie keineswegs unabänderlich gregeben. Denn die Vergangenheit enthält sowohl Philosophisches als auch Nichtphilosophisches in einem Neben- und Durcheinander und zur unabänderlichen, di. $h$. allgemeingültigen Unterscheidung des Philosophischen vom Nichtphilosophischen fehlt uns eben eine allgemeingültige Einsicht in bezug auf den allgemeinen Gegenstand der Philosophie selbst. So lange es uns an dieser letzteren gebricht, ist. Philosophiegeschichte als Wissenschaft ein pium desiderium. Soll das erste Problem der Philosophiegeschichte, das Problem ihrer Gegenstands-Setzung gelöst werden, so ist eine allgemeingütige Definition des Gegenstandes der Philosophie notwendig. Zwar gèht die hergebrachte Auffassung dahin, daß eine allgemeingültige Abgrenzung einer Eigenwirklichkeit der Philosophie eine Unmöglichkeit bedieute. Doch daß die letztere tatsächlich errungen werden können, daß̉ die Gefahren des Subjektivismus und die Verwicklungen dieses Historismus durch Anwendung der Methode, die Rudolf Eucken die noologische nennt, restlos überwunden werden können, das ist unsere Überzeugung, die wir in unserer Arbeit n. d. T. „Der Kampf um einen Gegenstand der Philosophie, Eine noologische Untersuchung" zu entwickeln suchen.

Auf diese Arbeit als Fortsetzung der vorliegenden Abhandlung sei hier verwiesen. Dort liegt ein Versuch einer ẹndgültigen Lösung für die Spannung der Aufgabe, die hier aufgerollt wurde. 